\title{
2 Das Kernkorpus: Briefe an den Zentralrat der Juden in Deutschland und die Israelische Botschaft in Berlin, 2002 bis 2012
}

\subsection{Zur Relevanz von Korpusanalysen und der Notwendigkeit einer Methodenerweiterung in der Antisemitismusforschung}

Bei unserer Untersuchung geht es um die Verbalmanifestationen der aktuellen Judenfeindschaft in Deutschland und die in diesen zum Ausdruck kommenden Denkstrukturen und Gefühle. Um empirisch fundierte Aussagen zu einem bestimmten sprachlichen oder kommunikativen Phänomen machen zu können, greift man in den Sprach-, Kommunikations- und Kognitionswissenschaften ${ }^{1}$ vermehrt auf Korpusanalysen zurück. Korpora sind Mengen von Texten, die $\mathrm{zu}$ einem bestimmten Thema über eine spezifische Zeitspanne erfasst wurden, um (über Einzelbeispiele hinausgehend) geeignete Vergleichsdaten quantitativ sowie qualitativ analysieren zu können (zur Korpusanalyse s. Scherer 2006: $36 \mathrm{f}$. und Bubenhofer 2009). Die Größenordnung kann je nach Bereich, Anlass oder Themenrelevanz variieren: Einige hundert Texte können ausreichend sein, um Grundmuster und deren Vorkommenshäufigkeiten $\mathrm{zu}$ erkennen, in der Regel handelt es sich aber um mehrere tausend oder sogar zehntausende Texte. Zeitlich kann sich die Korpuserhebung auf Tage, Monate oder (bei Langzeitstudien) auf Jahre erstrecken. So belief sich unsere Datenerhebung anlässlich der öffentlichen Debatte um den anti-israelischen Text von Günter Grass im Internet auf einige Tage, die zur Nahostberichterstattung in den Print- und Online-Medien dagegen auf mehrere Jahre und erfasste jeweils anlässlich bestimmter Krisensituationen in den Medien der Mitte publizierte Berichte und Kommentare. Der Fokus kann auf einem Medium und dort einem spezifischen Gebiet zu einem bestimmten Thema liegen (z. B. Online-Kommentare von Internetnutzern in Kommentarbereichen der Mainstreampresse im Berichtzeitraum zur Gaza-Flottillen-Aktion oder Äußerungen von Lesern in den Leserbrief-Rubriken der Printmedien zum Libanonkonflikt).

Bei dem unseren Textanalysen zugrunde gelegten Kernkorpus handelt es sich um eine Sammlung von über 14.000 E-Mails, Briefen, Postkarten und Faxen,

1 Von der Basisannahme ausgehend, dass sprachliche Äußerungen Spuren der kognitiven und emotionalen Aktivität der Sprachbenutzer sind, lassen sich entsprechend Rückschlüsse auf die Einstellungen der Verfasser der Texte ziehen (vgl. Kap. 3). 
die zwischen 2002 und 2012 an den Zentralrat der Juden in Deutschland und die Israelische Botschaft Berlin gesendet wurden (zu den genauen Angaben s. 2.2). Gemeinsam haben alle diese Texte, dass sie Diskursformen zum Thema ${ }^{2}$ Juden/ Judentum (in Deutschland) und/oder Israel sind, die von Sprachproduzenten aus dem deutschsprachigen Raum verfasst wurden und unaufgefordert an die beiden Institutionen geschickt wurden. Als Vergleichskorpus (das ebenfalls die Textsorte Brief/E-Mail umfasst) fungiert eine Menge von weit über 1.000 Texten, die 2004 an Michael Wolffsohn gesendet wurden (s. hierzu bereits Schwarz-Friesel 2007: 354) sowie eine Sammlung von 1.002 Zuschriften an die Israelischen Botschaften in Wien, Bern, Den Haag, Madrid, Brüssel, London, Dublin und Stockholm aus den Jahren 2010 bis 2011. Zudem wurden 29 Briefe bzw. E-Mails an die Journalistin des Hessischen Rundfunks, Esther Schapira, einbezogen, die diese als Reaktion auf den Film Drei Kugeln und ein totes Kind (ausgestrahlt am 18.03.2002 in der ARD-Reihe Das rote Quadrat) erhalten hatte. Vom Forum gegen Antisemitismus in Wien erhielten wir außerdem eine Übersicht zu judenfeindlichen Vorfällen mit einer Auswahl von repräsentativen Schmähbriefen, die zwischen 2000 und 2012 an jüdische Organisationen in Österreich geschickt wurden.

Es wurde keine spezifische (Vor-)Auswahl getroffen: Alle Schreiben (auch die positiven und solidarischen) sind berücksichtigt worden. Ergänzt werden diese Daten zusätzlich durch spezifische Subkorpora zu Texten aus dem öffentlichen Kommunikationsraum (vor allem Printmedien und Internetdiskurse), die dasselbe Thema zum Gegenstand haben und damit sowohl dem Vergleich als auch der Ergänzung unserer Untersuchungen dienen.

Der Abgleich mit diesen Textsorten zeigt, dass ähnliche Muster wiederkehren, weil sich die Mehrheit der Sprachproduzenten auch in (halb)öffentlichen Diskursen zu den jeweiligen Themen genauso äußert wie die Verfasser in den persönlichen E-Mails und Briefen. Weiterhin greifen wir mit der Untersuchung von Texten, die 2010 und 2011 an die Israelischen Botschaften in Österreich, der Schweiz, in Belgien, England, Irland, den Niederlanden, Schweden und Spanien geschickt wurden, auf ein Vergleichskorpus zurück, das kontrastive Analysen hinsichtlich des Anti-Israelismus in Europa zulässt.

Obgleich mittlerweile in vielen Disziplinen die korpusanalytische Methode benutzt wird, basieren die meisten Untersuchungen innerhalb der Antisemitismusforschung noch immer primär auf Einzelbeispielen (s. z. B. Benz 2004,

2 S. hierzu Busse/Teubert (1994: 14), die als Diskurs ein virtuelles Textkorpus sehen, dessen „Zusammensetzung durch im weitesten Sinne inhaltliche bzw. semantische Kriterien bestimmt wird“. Die zu einem bestimmten Korpus gehörenden Texte sind diejenigen, die sich aufgrund der Thematik mit einem spezifischen Diskursphänomen befassen und daher als repräsentativ für genau diesen Diskurs zu erachten sind. Vgl. hierzu auch Bubenhofer (2009). 
Rensmann 2004, Wistrich 2010). Die korpusbasierte Analyse ist einer auf unsystematisch ausgewählten Belegzitaten basierenden Herangehensweise jedoch überlegen, da sie durch ihre Datenvalidität stärker empirische Evidenz erbringt. Die Validität ergibt sich aus den großen Textmengen, die (zumeist elektronisch verfügbare) Korpora umfassen (vgl. Bubenhofer 2008: $407 \mathrm{ff}$. und 2009 sowie Stefanowitsch/Gries 2006). In den letzten zehn Jahren hat insbesondere die Korpuslinguistik ${ }^{3}$ gezeigt, wie durch elektronische Such- und statistische Berechnungsoperationen reliable Daten erhoben werden können, die bei unsystematischer Suche und Auswertung nicht hätten ermittelt werden können. Dabei ist jedoch zu betonen, dass eine quantitative Analyse im Bereich von Sprachgebrauchsmustern und Diskurseigenschaften die qualitative Inhaltsanalyse nicht ersetzen kann: Viele relevante Phänomene wie Implikaturen, Bewertungen und Stereotypausdruck lassen sich nur bei einer Detailanalyse, die Kontext und Spezifik des jeweiligen Textes berücksichtigt, und nur durch das Urteil eines menschlichen Betrachters erfassen. Erst die methodische Kombination von qualitativer kognitionslinguistischer Textanalyse mit der quantitativen, statistischen Korpusanalyse ermöglicht es, die Ergebnisse der Detailanalysen angemessen zu bewerten. Im Vordergrund der mikrostrukturellen Textanalyse steht das Herausarbeiten der spezifischen Verbalisierungsformen antisemitischer Stereotype und Argumentationsmuster. Die quantitative Analyse gibt Auskunft über die Häufigkeitsverteilung (generell und im zeitlichen Verlauf) von spezifischen antisemitischen Verbalisierungsformen.

Die quantitativ ausgerichteten Umfragen zu Juden und Judentum sowie Israel/Nahostkonflikt, die in der Soziologie und Psychologie turnusmäßig durchgeführt werden, können dagegen die Denk- und Gefühlsstrukturen der Befragten nicht oder nur sehr begrenzt erfassen, da sie stets mit sehr wenigen, vorformulierten und daher beeinflussenden Pauschalaussagen arbeiten (die aus semantisch begrenzten Werturteilen wie ,Juden haben in unserer Gesellschaft zu viel Einfluss“ oder „Israel ist ein aggressiver Staat“ bestehen). Verbalisierungsmuster werden gar nicht erfasst, da die Befragten lediglich ,ja“, „nein“ oder „weiß nicht“ ankreuzen oder sagen müssen.

Solche vorformulierten Aussagen können sogenannte Primingeffekte hervorrufen: Der Befragte wird unter Umständen erst durch die Rezeption des Satzes stimuliert, eine bestimmte Antwort zu geben. Faktoren wie soziale Erwünschtheit (Looking-good-Tendenz) können ebenfalls eine Rolle spielen (vgl. Brosius/

3 Die kritische Diskursanalyse betont ebenfalls seit Jahren die Relevanz von diskursorientierten Analysen, die textwissenschaftliche Methoden mit historischen und kulturwissenschaftlichen Untersuchungen verbinden (s. Wodak 1997 und Reisigl/Wodak 2001, Jäger ${ }^{3}$ 2001). 
Koschel 2001: 113f.). Es ist daher nicht zu entscheiden, ob die aus den Antworten ermittelten Einstellungen bereits bei den Befragten als permanente mentale Einstellungsmanifestation verankert waren oder ob sie durch die spezifische Aussage kontext- und situationsspezifisch aktiviert bzw. aktualisiert wurden. Siehe hierzu auch Scherer (2006: 2):

„Beide Methoden, die Sprecherbefragung wie auch das Experiment, haben aber den Nachteil, dass die Versuchspersonen vielleicht nicht die Antworten geben werden, die ihrem spontanen Sprachgebrauch entsprechen.“

Quantitative Studien $\mathrm{zu}$ antisemitischen Einstellungen in Deutschland (wie von Bergmann/Erb 1991, Heitmeyer 2002 bis 2012, Bergmann/Heitmeyer 2005b) berufen sich neben den Gütekriterien von Validität und Reliabilität des Erhebungsinstrumentes auf eine strukturgleiche Stichprobe aus der Gesamtpopulation. Über zufallsbasierte Auswahlverfahren sollen Stichproben gezogen werden, die die Bevölkerung in ihrer Gesamtheit repräsentieren. Umfang und Struktur der Stichprobe sind gerade bei Befragungen die zentralen Gütekriterien, obwohl aus logischen Gründen niemals die Merkmalsverteilungen in ihrer gesamten Vielzahl abgebildet werden können und die Redewendung von „repräsentativen Stichproben/Querschnitten“ somit auch stets nur eine Art Metapher ${ }^{4}$ ist (vgl. Diekmann 192008: 430).

Vor diesem Hintergrund muss hinsichtlich der Repräsentativität des korpusbasierten Untersuchungsmaterials Folgendes festgestellt werden:

Statistisch betrachtet lassen sich zur Repräsentativität des von uns untersuchten Korpus bezüglich der Gesamtbevölkerung keine Aussagen treffen. In der Regel beschränken sich die von den Schreibern gemachten Angaben auf Name und Adresse, häufig auch Alter und Beruf; sonstige demographische Daten sind aber unbekannt. Auf Basis der bekannten Angaben und der formalen und inhaltlichen Gestaltung der Zuschriften ist aber ersichtlich, dass das Korpus nicht auf (rechtsextreme oder anders geartete) Randgruppen oder von vornherein als antisemitisch klassifizierte Merkmalsträger begrenzt ist (wie z. B. bei Decker et al. 2006, Scherr/Schäuble 2007, Salzborn 2008). Rechtsextreme Schreiber sind mit

4 Dazu Diekmann ('192008: 432): „'Repräsentative’ Stichproben, vor allem Zufallsstrichproben, sind in der Praxis der Sozialforschung wichtig für bestimmte Zwecke: zur Schätzung von Verteilungen, also z. B. Anteils- oder Mittelwerten in der Population. Für andere Zwecke, wie die Prüfung allgemeiner Hypothesen, sind Repräsentativitätsproben meist entbehrlich. Wenn man dazu noch bedenkt, dass 'repräsentative Querschnitte' im Wortsinn nicht existieren, kann man mit einiger Berechtigung auch von einem Mythos der repräsentativen Stichprobe sprechen.“ Zu Fehlerquellen bei der Befragung s. ebenfalls Diekmann ( ${ }^{19} 2008$ : 446 ff.). Vgl. auch Brosius/ Koschel (2001: 122), Kirchhoff et al. (32003) und Porst (2008). 
elf Prozent (Kategorien 'rechts' und 'eher rechts' zusammen genommen) im Korpus vertreten, die Mehrheit bilden jedoch nicht sie, sondern Absender aus der politischen und sozioökonomischen Gesellschaftsmitte. So sind neben Schülern oder Angestellten auch viele Selbstständige, Beamte, Studenten und Akademiker vertreten. Viele der Absender thematisieren ihre politische Einstellung explizit und verorten sich selbst mehrheitlich in der Mitte oder geben sogar die Mitgliedschaft in einer der großen Volksparteien an. Auch hinsichtlich der geographischen Verteilung und der Altersangaben ist das Korpus ausgesprochen vielfältig. ${ }^{5}$

Nicht alle Deutschen sind in gleichem Maße motiviert, ihre Meinung zu Juden und Israel kundzutun (unabhängig davon, ob diese Meinung positiv, negativ oder gar antisemitisch ist). Der Grad der Betroffenheit bzw. des Bezugs $\mathrm{zu}$ wesentlichen Überzeugungen und Vorstellungen ist also für unser Korpus sehr hoch. Allerdings hat dies den Vorteil, dass das Kriterium der Zentralität ${ }^{6}$ auf jeden Fall erfüllt ist und Probleme mit „non opinions“ oder einem gleichgültigen Meinungsverhalten nicht vorliegen (vgl. Atteslander 122008: 61 f.). Damit ist anzunehmen, dass das untersuchte Material für die antisemitischen Konzepte und Stereotype derjenigen Deutschen, die antisemitisch eingestellt sind, Einblicke in typische Muster gestattet. ${ }^{7}$ Das Kriterium der „Repräsentativität“ bezieht sich bei der Korpusanalyse also auf den aktuellen antisemitischen Sprachgebrauch. Die Korpusdaten sind repräsentativ hinsichtlich der Sprachgebrauchsmuster im antisemitischen Diskurs, d. h. sie zeigen, welche Mittel und Strategien typisch für

5 Die Repräsentativität der Ergebnisse kann zudem dadurch erhöht werden, dass innerhalb des Gesamtkorpus einzelne Quotenstichproben gezogen werden bzw. nach sachlogischen Erwägungen eine bewusste Auswahl des Untersuchungsmaterials erfolgt (vgl. Brosius/Koschel 2001: 91). Dazu werden alle eher links oder eher rechts orientierten Zuschriften gefiltert, und aus innen wird eine eigene Stichprobe zusammengefasst und jeweils getrennt analysiert. Die Ergebnisse für diese Teilkorpora sind dann repräsentativ für die einzelnen Bevölkerungsgruppen wie Linksextreme, Rechtsextreme, Mitte usw. Durch eine solche Quotierung sind vergleichende Aussagen zwischen diesen Gruppen überhaupt erst möglich. Da dazu eine bewusste Einordnung in die Kategorien erfolgen muss, ist eine wahrscheinlichkeitstheoretische Prüfung der Ergebnisse jedoch nicht mehr möglich; die Erfahrung und Kenntnis des Forschers sind daher Indikator für die Zuverlässigkeit der Resultate (vgl. Atteslander ${ }^{12}$ 2008: 259).

6 Zentralität bezeichnet den Grad der Betroffenheit der Befragten bzw. den Bezug zu wesentlichen Überzeugungen und Vorstellungen (vgl. Atteslander ${ }^{12} 2008: 61 \mathrm{f}$.).

7 Einen besonderen Stellenwert haben bei der Analyse die Zeiträume, in denen relativ konstant besonders viele Zuschriften bei Zentralrat und Botschaft eingetroffen sind (2002, 2006, 2008/2009), weil mit der steigenden Zuschriftenzahl der Einfluss konfundierender Faktoren sinkt bzw. diese Faktoren eher normalverteilt sind. Zu den Aufmerksamkeitshöhepunkten sind zudem auch Personen bereit, sich zu äußern, die sonst eher eine geringe Motivation dazu verspüren oder eher moderate Einstellungen vertreten. 
judenfeindliche Sprache in Deutschland sind. Das bedeutet aber nicht, dass sie auch repräsentativ für die gesamte Kommunikation über Juden in Deutschland sind oder etwa Angaben über alle Deutschen machen.

Im Vergleich zu quantitativen Befragungsstudien ergeben sich zudem folgende Vorteile bei einer umfassenden Korpusanalyse: Es handelt sich bei den Zuschriften um selbstmotivierte Äußerungen und damit Versprachlichungen eigens gebildeter Bewusstseinsinhalte und nicht um Antworten auf vorformulierte Fragen, welche bestimmte Inhalte fokussieren oder auf einzelne Aspekte einschränken. Damit geht unser Ansatz über die Erfassung der spontanen Einstellungsebene hinaus und erhebt Informationen zu mental stabilen Denk- und Gefühlswerten zu Juden/Judentum und Israel. Eine Beeinflussung über die Frageformulierung ist ausgeschlossen; und es kommt nicht $\mathrm{zu}$ inhaltlichen oder emotionalen Ausstrahlungseffekten von Frage zu Frage, wie sie z. B. bei den Fragebögen Heitmeyers u. a. wegen der Fülle und Nähe vorurteilsbehafteter Fragen wahrscheinlich sind (vgl. Brosius/Koschel 2001: 122, Kirchhoff et al. ${ }^{32} 2003$, Porst 2008). Auch entfallen eventuell unerwünschte Effekte, die bei Befragungen als reaktive Methode auftreten, wie z. B. Tendenzen der sozialen Erwünschtheit bzw. des Looking-good-Effekts oder Versuchsleitereffekte (vgl. Brosius/Koschel 2001: 146 ff., Diekmann ${ }^{19} 2008$ : 446 ff.).

Eine Diskrepanz zwischen einer in einer Befragung ermittelten Einstellung und dem tatsächlichen Verhalten eines Probanden, wie sie bei Interviews und Befragungen auftreten kann (vgl. Atteslander ${ }^{12} 2008$ : 161), tritt bei der Analyse von Zuschriften an den ZJD und die IBD ebenfalls nicht auf. Bei den sprachlichen Äußerungen handelt es sich um manifestierte Einstellungen, weil sprachliche Äußerungen in Form einer adressierten Zuschrift Handlungen sind und zugleich Ausdruck einer emotionalen und kognitiven Haltung.

Die meisten Umfragen werden der Komplexität des Phänomens Antisemitismus nicht gerecht, ${ }^{8}$ d.h. eine differenzierte Befragung, die israel-kritische Haltungen und Antisemitismus sowie dessen verschiedene Ausprägungen berücksichtigt, findet nicht statt.

Zwar stellen empirische Umfragen wichtige Methoden zur Feststellung gesamtgesellschaftlicher Tendenzen dar, doch tiefergehende Analysen oder

\footnotetext{
8 Sie können es wohl auch nicht, weil es gar nicht möglich ist, eine große Anzahl an Personen (die für eine repräsentative Stichprobe nötig wäre) in einer kurzen Befragungssituation mit präzisen Detailfragen zu Judentum, Geschichtsbild und Israel zu konfrontieren bzw. diese Fragen zu standardisieren. Zudem zeichnet sich bei Befragungsmethoden ab, dass Lernprozesse und Reflexionen der Probanden dazu führen, ihnen gestellte Fragen im Laufe der Befragung kritischer zu sehen und vorsichtig(er) zu beantworten. Dies schränkt Validität und Reliabilität von Befragungen generell ein.
} 
gar Erklärungen erlauben sie nicht. Sie decken keine mentalen Verknüpfungen zwischen Stereotypen auf, sie geben keine Auskunft darüber, wie über verbale Mittel und Strategien Denk- und Gefühlsstrukturen der Judenfeindschaft tradiert werden, und sie geben keinen Einblick in die personenbezogenen Einstellungsmuster und Weltbilder.

Die sozialwissenschaftlichen Studien müssen daher um kognitionswissenschaftliche Analysen individueller Einstellungsrepräsentationen ergänzt werden, die sich aus den spontanen Verbalisierungsmustern erschließen lassen. Nur so können wir die tatsächlichen mentalen Repräsentationen zu Juden und Israel eruieren und eine eventuelle Kontinuität bzw. Veränderung von Stereotypen und Klischees postulieren. Die aktuelle Antisemitismusforschung muss sich also methodisch öffnen und neben den üblichen, „konservativen“ Methoden der sozialwissenschaftlichen Befragung und der historischen Deskription von Einzelereignissen (interdisziplinäre) Korpusanalysen und text- wie kognitionslinguistische Detailanalysen einbeziehen, will sie dem Anspruch gerecht werden, Judenfeindschaft in ihren diversen Facetten und Erscheinungsformen inhaltlich angemessen und repräsentativ zu erfassen. Vgl. hierzu auch Rensmann/Schoeps (2008: 31): „[...] es braucht vielmehr ein breites Set von diskursanalytischen und diskurshistorischen Zugängen [...].“

\subsection{Spezifika des Korpus}

\section{Umfang des Korpus, Subkorpora und Stichprobenanalysen}

Das Kernkorpus unserer Untersuchung besteht aus über 14.000 Zuschriften in Form von Briefen, E-Mails oder Faxen, die in den Jahren 2002 bis 2012 beim Zentralrat der Juden in Deutschland (vom 31.03.2002 bis 24.07.2009) und der Israelischen Botschaft in Deutschland (vom 17.10.2003 bis 10.04.2012) eingingen. Die Schreiben sind in der Regel an die damalige Vorsitzende des Zentralrats Charlotte Knobloch (und zuvor an Paul Spiegel) und den israelischen Botschafter Yoram Ben-Zeev (zuvor Shimon Stein) adressiert, zum Teil aber auch ohne Anrede lediglich an die Institutionen abgeschickt. Das von uns katalogisierte Material umfasst in den Jahren 2002 bis Anfang 2012 insgesamt 14.003 Zuschriften an die beiden Institutionen. 


\section{Themenschwerpunkte bzw. Zusammenhang mit relevanten Diskursereignissen}

Die Zahl der Zuschriften fluktuiert zwischen 2002 und 2005 stark, dies liegt zum einen an der Bereitstellung durch die beiden Institutionen, ${ }^{9}$ zum anderen an der unterschiedlichen Aufmerksamkeit, die den zentralen politischen Diskursereignissen gewidmet wird. Ab dem Jahr 2006 ist das Material nach Aussage der beiden Institutionen jedoch lückenlos an unsere Forschergruppe übermittelt worden. Die auftretenden Schwankungen sind demnach durch diskursive Verschiebungen bedingt und aufschlussreich für die quantitative Analyse. Etwa zwei Drittel des Materials stammen von der Israelischen Botschaft, sie erhielt in den Jahren 2006 bis 2009 mehr Zuschriften als der Zentralrat. So gingen 2006 anlässlich des Libanonkrieges beim Zentralrat 1.170, bei der Botschaft 2.100 Schreiben ein; 2009 waren es anlässlich der Operation „Gegossenes Blei“ nochmals doppelt so viele Zuschriften. Der Zentralrat hingegen sticht im Jahr 2002 hervor, als er im Zuge der Möllemann-Friedman-Debatte ${ }^{10}$ im Zentrum der Aufmerksamkeit steht.

Als Themenanlässe fungieren in der Regel Verlautbarungen des Zentralrates oder mediale Berichte über politische Entwicklungen in Israel bzw. im israelischpalästinensischen Konflikt. Im Jahr 2006 ist es der Libanonkrieg, 2007 die Eskalation im Gazastreifen. Allein in den Sommermonaten des Jahres 2006 trafen knapp 2.000 Zuschriften bei der Botschaft ein, beim Zentralrat über 1.000, bei beiden Institutionen bildet der Juli das Maximum (Botschaft Juli $n=1.155$; vgl. Abbildung 1).

9 Bis 2006 stellten Botschaft und Zentralrat die Zuschriften sporadisch zur Verfügung, Rückantworten fehlen zumeist, Attachments sind oft unvollständig. Die starke Fluktuation spricht dafür, dass beide Institutionen nicht alle Zuschriften systematisch sammeln, sondern ggf. auch nach eigenen Kriterien archivieren (so z. B. die Kommentierung „Hassmails“ oder „Soli“). Des Weiteren finden sich einige hundert Briefe an die Israelische Botschaft und ca. 200 Briefe an den Zentralrat der Juden in Deutschland des Zeitraums 2000 bis 2003 im Archiv des Zentrums für Antisemitismusforschung in Berlin. Der Zentralrat stellte ferner Samuel Salzborn mehrere Hundert Zuschriften zur Verfügung, die in den Wochen und Monaten nach Kardinal Meisners Predigt im Januar 2005 eingingen (vgl. Salzborn 2005: 919).

10 Die Zuschriften zur Möllemann-Friedman-Auseinandersetzung aus dem Jahr 2002 beschränken sich auf den Zentralrat der Juden. Ob und in welcher Größenordnung die IBD Schreiben erhielt, konnte nicht mehr rekonstruiert werden. 


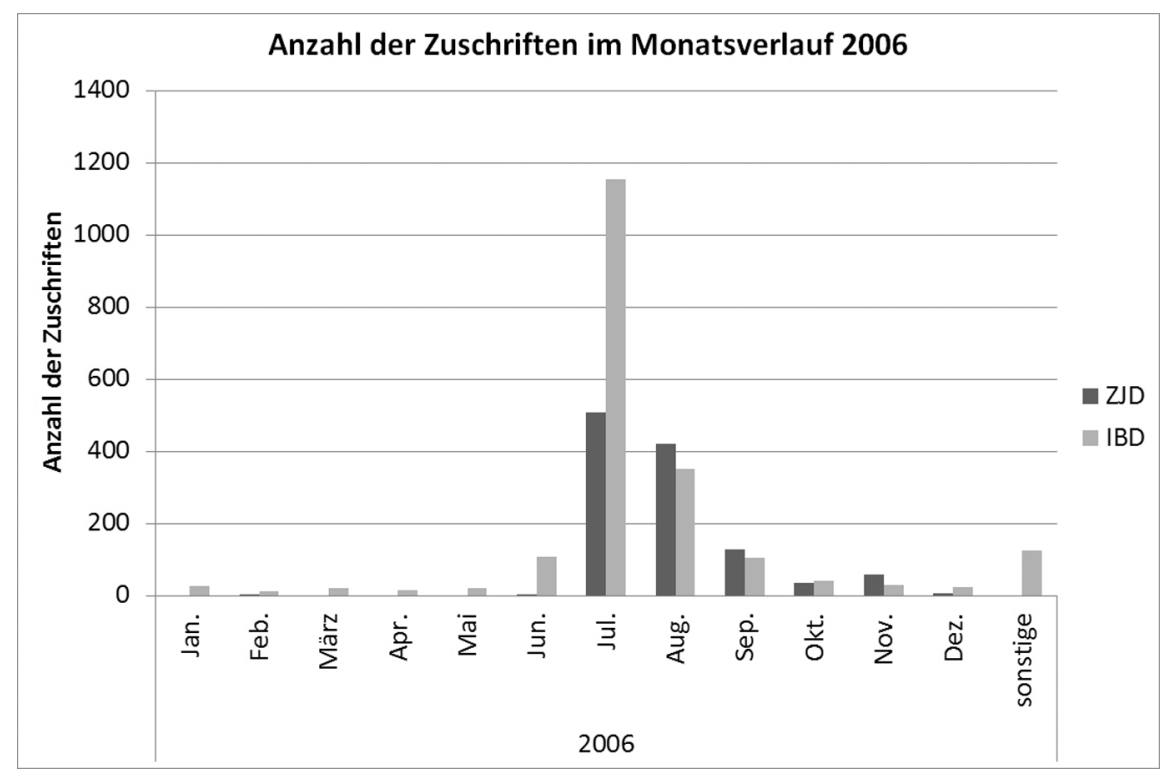

Abbildung 1: Zuschriften im Monatsverlauf 2006.

Auffällig ist, dass sich von Juli bis September 2006 eine große Zahl an Schreibern an den Zentralrat der Juden in Deutschland wendet $(n=1.067)$ und diesen wegen der Situation im Libanon zum Einlenken auffordert. Diese Schreiber sehen demnach einen Handlungsbedarf auf Seiten des Zentralrats, wenn es um die Außenpolitik oder das militärische Vorgehen des Staates Israel geht. Dies mag zum Teil auf die Solidaritätsaufforderungen des Zentralrats zurückzuführen sein; ${ }^{11}$ es deutet aber stark darauf hin, dass Juden in Deutschland für die Politik des israelischen Staates verantwortlich gemacht werden. Des Weiteren ist hierin ein Indiz für die konzeptuelle Verschmelzung der Konzepte JUDE(N) und ISRAELI(s) zu sehen (s. hierzu ausführlich die Kap. 5.2 und 7). Im Konflikt zwischen der Hisbollah und Israel im Libanon im Jahr 2006 stehen die Äußerungen der Entwicklungshilfeministerin Heidemarie Wieczorek-Zeul wochenlang im Mittelpunkt des Interesses. Deren Kritik an von Israel verwendeten Streubomben wird in der Mehrzahl der Briefe aufgenommen. In diesem Zusammenhang wird dem Zentralrat eine parteiische Sicht auf den Nahostkonflikt vorgeworfen und seine Solidaritätsaufrufe für Israel werden abgelehnt. Über das gesamte Jahr 2007

11 Der ZJD rief 2006 mehrfach zur Solidarität mit Israel und den entführten Soldaten auf, so z. B. am 19. Juli 2006 (vgl. http://www.zentralratdjuden.de/de/article/1060.html; letzter Zugriff am 02.09.2012). 
bleiben die Zuschriften an die IBD relativ konstant bei ca. 100 pro Monat, beim Zentralrat gehen pro Monat zwischen 10 und 20 Schreiben ein; eine Ausnahme bildet beim ZJD der Monat April.

Der Gaza-Konflikt führt zum Jahreswechsel 2008/2009 zu einem neuen Anstieg: Bei der Botschaft gehen 4.028 Schreiben, beim Zentralrat 867 Texte ein. Insgesamt spiegelt sich im Zuschriftenaufkommen die mediale Aufmerksamkeit, die den jeweiligen Eskalationsphasen in Deutschland gewidmet wird, wider; je ausführlicher und kontroverser das Geschehen in Nahost in den Medien dargestellt und in der (internationalen und deutschen) Politik debattiert wird, desto mehr Zuschriften erhalten Botschaft und Zentralrat (s. Abbildung 1). In der Regel schreiben die Verfasser ihre Texte unmittelbar nach Veröffentlichung einer Äußerung vom ZJD oder einer medialen Sendung: Einer Art Reiz-Reaktions-Schema folgend äußern sich die Schreiber entweder sofort nach der Rezeption der Äußerung oder am darauffolgenden Tag. Seit 2006 sind Medienberichte der ausschlaggebende Anlass für die Schreiber. Häufig sind diese Berichte oder Pressemeldungen als Attachments angefügt oder verlinkt bzw. es wird explizit auf diese Texte verwiesen (s. hierzu Kap. 7.3). Zudem nimmt die Anzahl angehängter Bilder und PDF-Dateien im Verlauf von 2002 bis 2012 stark zu, ebenso steigt die Zahl von Sammel-E-Mails, Petitionen und Unterschriftenlisten.

Das 60. Staatsjubiläum Israels bildet im Mai 2008 den Hauptanlass für Zuschriften, hier ist zum ersten Mal im gesamten Korpus ein hoher Anteil solidarischer E-Mails und vor allem von Glückwunschschreiben zu verzeichnen. Ein ähnlicher Trend zeichnet sich im Zusammenhang mit dem Gefangenenaustausch zwischen Israel und der Hisbollah ab, bei dem die Leichname von Eldad Regev und Ehud Goldwasser an Israel übergeben wurden. Hier sind viele Solidaritätsbekundungen mit den Angehörigen der Soldaten, aber auch mit dem Staat Israel allgemein zu konstatieren. Mit diesen Zuschriften wenden sich nun auch vermehrt Personen, die Israel gegenüber positiv eingestellt sind, an die Botschaft, welche das Sicherheitsbedürfnis und die Gefährdung des Staates Israel ansprechen (und sich zum Teil auch medienkritisch gegen anti-israelische oder monoperspektivierte Berichterstattung aussprechen).

Andererseits ist jedoch in den israel-kritischen und antisemitischen Zuschriften zugleich ein deutlicher Anstieg expliziter und sehr aggressiver Hassmails zu verzeichnen. Immer mehr Schreiber benutzen seit Anfang 2009 (Gaza-Konflikt) brachiale Sprachgebrauchsmuster, ${ }^{12}$ Beleidigungen, Beschimpfungen, direkte

12 Die Agentur für Grundrechte der Europäischen Union (FRA) spricht von einer Zunahme des Antisemitismus im Zuge des Gaza-Konflikts (vgl. FRA 2009). Die vom Bundesinnenministerium im April 2009 veröffentlichten Zahlen zu antisemitischen Straftaten werden von unseren Befunden ebenfalls dahingehend bestätigt, dass eine Radikalisierung der Ausdrucksformen 
Drohungen und Verwünschungen nehmen zu (s. hierzu Kap.10.1). Auffällig oft werden nun auch explizite NS-Vergleiche gezogen. Auch die Ausdrucksformen von Schreibern aus der Mitte der Gesellschaft radikalisieren sich zunehmend. Die Berichterstattung zum Stopp der türkischen Gaza-Flottille durch die israelische Armee Ende Mai 2010 führt zu einer Flut von 830 Schreiben an die Botschaft, die sich mehrheitlich durch drastische Verbal-Aggressivitäten auszeichnen.

\section{Katalogisierung, Stichprobenumfang und Subkorpora}

Jede einzelne der 14.003 Zuschriften wurde bis Ende 2011 mit einer Sigle der Art [ZJD_29.05.2007_Sch_002] versehen, um die Zitierweise einheitlich und übersichtlich gestalten zu können sowie die Identifizierbarkeit/Auffindbarkeit im Gesamtkorpus zu gewährleisten. Das Kürzel gibt einige grundlegende Informationen an: ZJD steht für Zentralrat der Juden in Deutschland, mit IBD beginnende Kürzel hingegen markieren Zuschriften, die bei der Israelischen Botschaft in Deutschland eingegangen sind. Das Datum gibt an, an welchem Tag der ZJD oder die IBD die Zuschrift erhielt; für den Namen des Schreibers bzw. der Schreiberin wurde ein anonymisierendes Kürzel der ersten zwei bzw. drei Buchstaben eingesetzt. 001 und 002 geben an, dass es sich um die erste oder zweite Zuschrift desselben Sprachproduzenten handelt. So können Mehrfachschreiber identifiziert werden.

Eine detaillierte Klassifikation und Einzelanalyse aller 14.003 Zuschriften nach den relevanten formalen und inhaltlichen Kategorien (s. das Variablenset) war nicht möglich, insbesondere nach dem exorbitanten Anstieg an Zuschriften ab 2009. Hier spielt auch der Umfang der Zuschriften eine Rolle, zur quantifizierenden Analyse müssen die häufig mehrseitigen Texte stets komplett gelesen werden. Um dennoch quantitative Angaben machen zu können, wurde eine repräsentative Stichprobe erstellt: Hierfür wurde der Zeitraum 01.01.2002 bis 31.12.2007 ausgewählt. Innerhalb dieses Zeitraums liegen 4.912 Zuschriften, die sich auf für den Zentralrat und die Israelische Botschaft relevante Ereignisse beziehen. Der gewählte Zeitraum deckt also eine Vielzahl relevanter Themenanlässe ab und ist groß genug, um Veränderungen im Jahresverlauf nachzeich-

stattfindet. Das BMI konstatierte ein Rekordhoch der politisch motivierten Straftaten seit Beginn der Erhebung 2001, insgesamt wurden über 31.000 Delikte verzeichnet. Einen starken Anstieg gab es bei rechtsextremen Straftaten (plus 16 Prozent) auf 20.422. Dazu hätten 2008 vor allem von unbekannten Tätern verübte „Propagandadelikte“ (der NS-Verherrlichung) beigetragen, diese Propagandadelikte machten mittlerweile 69,9 Prozent aller rechten Straftaten aus. 
nen zu können. Zu beachten ist auch, dass mit dem Anstieg im Jahr 2009 eine große Menge sehr homogenen Materials eingegangen ist, es handelt sich dabei fast ausschließlich um E-Mails. Deren Schreibanlässe bilden stets die israelische Politik oder Militäreinsätze. Das Material, welches ab 2008 eingegangen ist, wurde daher stichprobenartig auf Auffälligkeiten oder Veränderungen geprüft. Wenn spezifische Verbalisierungen oder argumentative Muster oder besondere Neuerungen auffielen, wurde deren quantitative Relevanz mittels entsprechender Stichwortsuche oder kursorischen Lesens geschätzt. Die Analysen wurden durch linguistisch geschulte Kodierer vorgenommen, die über die entsprechenden Kriterien informiert waren (also kein Blind-Kodieren). Über die Analyse und Kategorisierung prototypischer Fälle sowie Diskussion problematischer Zuschriften waren die Kodierer entsprechend geschult (Probekodierungen wurden bereits während der explorativen Phase durchgeführt). Während des Kodierprozesses ${ }^{13}$ wurden unabhängige Doppelkodierungen vorgenommen und dann in der Forschergruppe abgeglichen.

In die qualitativen Einzelanalysen wurden Textbeispiele auch des Materials aus den Jahren 2009 bis 2012 mit einbezogen, um darzustellen, dass die hier skizzierten Veränderungen nur wenige Einzelaspekte betreffen und die meisten verbalisierten Stereotype und textuellen Strategien fortbestehen und sich seit 2006/2007 wenig verändert haben. Die Ergebnisse, die sich auf die quantitativ klassifizierte Stichprobe ${ }^{14}$ beziehen, sind daher für die Grundgesamtheit der Daten generalisierbar. Aus der Stichprobenerhebung zu den 4.912 Zuschriften und deren Inhaltsanalysen lassen sich folgende Tendenzen hinsichtlich der politischen Ausrichtung der Schreiber feststellen:

13 Es wurde immer konservativ kodiert: War also eine unklare Tendenz oder eine graduelle Abstufung in der Analyseeinheit Zuschrift abzuwägen, wurde jeweils die geringere Variablenausprägung gewählt.

14 Als Vergleichsinformation: Die Forsa-Umfragen, die Trends in der Gesamtbevölkerung von 82 Millionen Deutschen erheben wollen, basieren in der Regel auf 2.000 bis 4.000 Befragungen. Die Heitmeyer-Studie Deutsche Zustände involviert in der Haupterhebung 2.000 Personen und über die Jahre 23.000 . 


\section{Zuschriften nach politischer Tendenz}

$\begin{array}{lc}\text { rechtsextrem (4\%) } & \text { eher rechts (8\%) } \\ \text { eher links (13\%) } & \text { Mitte }(65 \%)\end{array}$

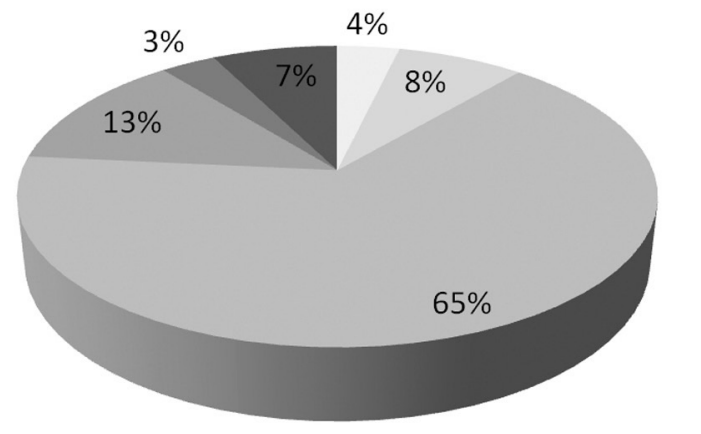

Abbildung 2: Verteilung der Zuschriften nach politischem Spektrum.

Die klare Mehrheit der Schreiber ist der politischen Mitte ${ }^{15}$ zuzuordnen $(n=3.204$, $65 \%$ ), dies belegt die Relevanz des Themas „Antisemitismus - ein Phänomen der Mitte“ (s. Schwarz-Friesel et al. 2010).

Das Konzept der Mitte prägt unsere Vorstellung von politischer Normalität und Stabilität (vgl. Heitmeyer 2007: 27); die Mitte wird in der Regel als eine Positionierung jenseits extremistischer und antisemitischer Einstellungen verstanden bzw. gilt als von diesen Rändern abgrenzbar (vgl. z. B. Backes 1989, Backes/Jesse 1989). Die Projektanalysen zeigen aber, dass Meinungen, die in solche Extrempositionen übergehen, auch in breiten gesellschaftlichen Schichten bzw. in der Mitte oder Mehrheit vertreten werden.

Abiturienten, Studierende, Anwälte, Journalisten, Ärzte, Pfarrer, Selbständige und Lokalpolitiker, aber auch Professoren artikulieren in ihren Schreiben zahlreiche Stereotype (s. hierzu Kap. 5). Von den Schreibern geben neun Prozent (bei Schreiben an die IBD) bzw. sieben Prozent (bei Texten an den ZJD) einen aka-

15 Politische und soziale Mitte sind häufig nicht deckungsgleich (vgl. Heitmeyer 2007: 28); die Definition nach sozioökonomischen Merkmalen ist daher für unsere Analyse sinnvoller. Typischerweise zählen zur Mitte der Gesellschaft insgesamt politisch nicht radikale, ökonomisch gut situierte und gebildete Bürger. Vgl. Hradil/Schmidt (2007: 169 f.) und Nolte/ Hilpert (2007: 31 ff.). Zum Phänomen und Begriff der „Mitte“ s. auch Rensmann (2004: 104) und Zick/Küpper (2006b: 115): „Die Mitte ist vielschichtig und mehrdimensional. Bei der Analyse sozialer Vorurteile erscheint sie primär als politische Position.“ 
demischen Titel an, haben also mindestens ein Hochschulstudium abgeschlossen. Hier wird ersichtlich, dass sich auch gesellschaftliche Eliten und Hochgebildete (erkennbar z.B. an genannten Berufsbezeichnungen) mit antisemitischen Äußerungen an die IBD und den ZJD wenden.

Rechtsextreme und linksextreme Zuschriften halten sich in etwa die Waage und kommen mit jeweils unter vier Prozent überraschend selten vor. Zuschriften, die als „eher links“ klassifiziert werden können, kommen fast doppelt so oft vor wie Zuschriften „eher rechter/rechtskonservativer Schreiber“ (für „eher rechts“ $\mathrm{n}=7,6 \%$; für „eher links“ $\mathrm{n}=12,7 \%$ ). Der Anteil der Rechtsextremen bzw. eher rechten Schreiber ist beim Zentralrat doppelt so hoch wie bei der Botschaft, was vermutlich am Themenhorizont des Zentralratskorpus liegt; Rechtsextreme vertreten häufiger klassische Stereotype und Einstellungen und weniger neue israelbezogene Vorurteile, daher wenden sie sich eher an den Zentralrat als an die Botschaft. Die Zahl der Zuschriften Linksextremer ist bei der Botschaft höher als beim Zentralrat; sie kommunizieren ihre israelfeindlichen oder israel-kritischen Positionen eher an die Botschaft, weil sie zum Teil dezidiert eine Differenzierung zwischen Judentum und Israel vornehmen bzw. sich als antizionistisch und antiantisemitisch definieren.

Klar ersichtlich ist, dass keinesfalls die Zuschriften aus extremen Randgruppen das Schwergewicht des Korpus bilden. Vielmehr sind Menschen der sogenannten Gesellschaftsmitte in hohem Maße motiviert, sich an den Zentralrat oder die Botschaft zu wenden. Das Aufkommen rechts- oder linksextremer Zuschriften beim ZJD scheint zu dem Personenpotenzial des Links- bzw. Rechtsextremismus in Deutschland, welches die Verfassungsschutzberichte jährlich ausweisen, äquivalent zu sein. ${ }^{16}$ Die Zahlen decken sich auch in etwa mit den von Zick/Küpper (2006b: 118) erfragten politischen Selbsteinschätzungen: Demnach bezeichnen sich im Jahr 2005 sieben Prozent der Befragten als links, 22 Prozent als eher links, 13 Prozent als eher rechts und knapp vier Prozent als rechts, während sich 54 Prozent genau der Mitte zuordnen (vgl. auch Hradil/Schmidt 2007: 174).

Der Residualkategorie (ambige bzw. nicht klar klassifizierbare, islamistische ${ }^{17}$ oder psychopathologische Zuschriften) gehören 368 Zuschriften an (zusammen 7,5 Prozent). Der Anteil der Schreiber mit islamistischem Hintergrund ist bei der Botschaft höher als beim ZJD (dabei sind eigene Gruppenidentität, Solidarisie-

16 Zum rechtsextremen Personenpotenzial in Parteien und freien Kameradschaften vgl. http:// www.verfassungsschutz.de/de/arbeitsfelder/af_rechtsextremismus/; zur Bedeutung des Linksextremismus in Deutschland siehe http://www.verfassungsschutz.de/de/arbeitsfelder/ af_linksextremismus/; letzter Zugriff jeweils am 02.09.2012.

17 Von Islamisten oder Exil-Palästinensern abgesendete Schreiben sind von so geringer Zahl, dass eine gesonderte Aufführung nicht indiziert ist. 
rung mit den „palästinensischen Brüdern“ bzw. eine Art „Religionssolidarität“ relevant), insgesamt aber zu vernachlässigen. Bei den muslimischen Schreibern stellt eventuell auch ein Mangel an Sprachkompetenz ein Hemmnis dar, weshalb deren Prozentsatz so gering ist.

\begin{tabular}{lrrrrrr} 
& & ZJD & & IBD & & gesamt \\
& $\mathrm{n}$ & Prozent & $\mathrm{n}$ & Prozent & $\mathrm{n}$ & Prozent \\
\hline Mitte & 1418 & $65,3 \%$ & 1786 & $65,2 \%$ & 3204 & $65,2 \%$ \\
eher links & 244 & $11,2 \%$ & 379 & $13,8 \%$ & 623 & $12,7 \%$ \\
linksextrem & 37 & $1,7 \%$ & 125 & $4,6 \%$ & 162 & $3,3 \%$ \\
eher rechts & 247 & $11,4 \%$ & 126 & $4,6 \%$ & 373 & $7,6 \%$ \\
rechtsextrem & 106 & $4,9 \%$ & 76 & $2,8 \%$ & 182 & $3,7 \%$ \\
islamistisch & 23 & $1,1 \%$ & 106 & $3,9 \%$ & 129 & $2,6 \%$ \\
psych. auffällig & 14 & $0,6 \%$ & 71 & $2,6 \%$ & 85 & $1,7 \%$ \\
sonstige & 82 & $3,8 \%$ & 72 & $2,6 \%$ & 154 & $3,1 \%$ \\
\hline & & & 2741 & & 4912 & \\
gesamt & 2171 & & & & &
\end{tabular}

Tabelle 1: Anzahl der Zuschriften nach politischer Tendenz.

In Bezug auf die soziale Schichtung und die geographische Verteilung ergeben sich kaum Auffälligkeiten. Die Zuschriften verteilen sich relativ gleichmäßig auf Ost- und Westdeutschland sowie Städte und ländliche Regionen.

Es wurden spezifische Subkorpora innerhalb des Gesamtkorpus gesondert analysiert, um kontrastive Analysen bei Intergruppenvergleichen deutlicher zu erfassen: z. B. Sprachgebrauchsmuster bei Extremisten und bei Verfassern aus der Mitte, Unterschiede zwischen Zuschriften an den ZJD und die IBD, Zuschriften anlässlich der Möllemann-Friedman-Debatte. Als interessantes Teilkorpus wurden auch die Zuschriften herausgegriffen, die sich auf die Trauerrede des baden-württembergischen Ministerpräsidenten Günther Oettinger für Hans Filbinger bzw. auf die Kritik des Zentralrats der Juden an dieser Rede beziehen, da hier thematisch ein unmittelbarer Bezug zur deutschen NS-Vergangenheit gegeben ist. Auch ist ein Vergleich im Zeitverlauf nach Jahren interessant, weil so veränderte Kommunikationsstrukturen ablesbar sind (z. B. hinsichtlich der Verwendung von NS-Vergleichen oder der Abnahme indirekter Sprechakte zugunsten direkter Sprachhandlungen sowie der Artikulation klassischer judenfeindlicher Stereotype).

Um Wörter, die mit einem bestimmten anderen Wort signifikant häufig in bestimmten Kontexten erscheinen, also Kollokationen (im Sinne von Häufung benutzter Wortverbindungen) bilden, erfassen zu können, wurden zu Subkorpora Kookkurrenz- bzw. Kollokationsanalysen durchgeführt (vgl. Perkuhn/Belica 
2004). Zusätzlich wurden anhand von Stichproben (die in der Regel um die 1.000 Texte erfassen) Charakteristika von auffälligen Wortfeldern bzw. semantischen Feldern untersucht (vgl. Schwarz/Chur ${ }^{52007:} 60$ f.).

\section{Zuschriftenart und Anonymität}

Bei den vorliegenden Zuschriftenarten ergibt sich für Zentralrat und Botschaft zwischen 2002 und 2007 ein einheitliches Bild: Bei beiden überwiegen E-Mails (rund 65 Prozent), gefolgt von Briefsendungen (inklusive Postkarten knapp 28 Prozent), am seltensten werden Telefaxe (jeweils unter 10 Prozent) geschickt. In der gewählten Zuschriftenart vollzieht sich über die Jahre hinweg ein Wandel: Während im Jahr 2002 noch klassische Briefsendungen überwiegen, werden in den Jahren 2006/2007 mehrheitlich E-Mails versendet. Die schnelle und kostengünstige Kommunikationsform (die seit 2008 die mit geschätzten 90 Prozent dominante Informationsvermittlung darstellt) bietet die Möglichkeit, sich schnell und unkompliziert (ggf. unverbindlich und anonym) an die Institutionen zu wenden.

\begin{tabular}{lrrrrrr} 
Zuschriftenart & & ZJD & & IBD & & gesamt \\
& $n$ & Prozent & $\mathrm{n}$ & Prozent & $\mathrm{n}$ & Prozent \\
\hline Briefe (inkl. P/Ph) & 599 & $27,6 \%$ & 755 & $27,5 \%$ & 1354 & $27,6 \%$ \\
Faxe & 171 & $7,9 \%$ & 159 & $5,8 \%$ & 330 & $6,7 \%$ \\
E-Mails & 1401 & $64,5 \%$ & 1827 & $66,7 \%$ & 3228 & $65,7 \%$ \\
& 2171 & & 2741 & & 4912 & \\
\hline
\end{tabular}

Tabelle 2: Art der Zuschrift.

Die meisten Zuschriften werden jedoch mit Namen und Adresse eingeschickt, nur etwa 13 Prozent der Nachrichten werden anonym versendet (bei IBD und ZJD). Bei 41 Prozent (ZJD) bzw. 37 Prozent (IBD) der Zusendungen ist neben dem Namen auch die vollständige Postanschrift, unter Umständen auch die Telefonnummer oder eine Homepage angegeben. Bei den E-Mails überwiegen aber Zuschriften, bei denen neben dem Namen nur der Wohnort (und natürlich die E-Mail-Adresse) angegeben werden. E-Mails sind diejenigen Zuschriften, die am häufigsten anonym verfasst sind. Im Zeitraum 2002 bis 2007 schwankt der Prozentsatz von anonymen Absendern im Jahresverlauf zwischen 9 und 15 Prozent. Es gibt quantitativ keinen klaren Trend zu einer Zu- oder Abnahme von anonym versendeten Texten. Dagegen ist eindeutig zu konstatieren, dass sich insgesamt mehr Men- 
schen mit Namen und Adresse an den ZJD oder die IBD wenden und dennoch (zum Teil extreme Formen von) Verbal-Antisemitismus artikulieren.

Interessant ist die Verteilung der anonymen Zuschriften im politischen Rechts-Mitte-Links-Spektrum. Die als gesellschaftliche oder politische Mitte kategorisierten Zuschriften sind am seltensten anonym verfasst. Die Mitte ist am wenigsten geneigt bzw. sieht es am wenigsten für nötig an, Angaben zur eigenen Person geheim zu halten. Diese Schreiber halten ihre Meinung also für öffentlich sagbar/vertretbar (und würden dies wahrscheinlich auch in anderer Form als einer Briefsendung oder E-Mail an den ZJD oder die IBD tun). Dieser Befund korrespondiert mit der häufigen Thematisierung der eigenen Integrität und dem Phänomen der individuellen Antisemitismus-Abwehr; diese Menschen begreifen sich selbst bzw. ihre Meinung nicht als antisemitisch oder problematisch, sie sehen ihre Standpunkte als notwendig und berechtigt an und bürgen dafür mit ihrem Namen.

Davon heben sich die extremistischen Schreiber ${ }^{18}$ deutlich ab, sowohl unter Rechts- als auch Linksextremen (bzw. deren abgeschwächten Varianten „eher links“/,eher rechts“) sind die Anonymitätswerte zweistellig, d.h. bei diesen Gruppen schätzen wesentlich mehr Schreiber ihre Äußerungen als so problematisch ein, dass sie ihren Namen lieber nicht nennen (sei es aus Angst vor vermuteten Sanktionen, was zum Teil in absurde Hinweise auf die angeblichen Tätigkeiten des israelischen Geheimdienstes ausufert). Den höchsten Anonymitätswert weisen die Rechtsextremen auf; fast 43 Prozent der Rechtsextremen (für die Botschaft allein betrachtet betrifft es sogar die Hälfte aller rechtsextremen Absender) schreiben anonym. Das ist ein deutliches Indiz dafür, dass diese Personen ihre Ansichten und Meinungen als nicht sagbar oder konsensfähig erachten. Hier deckt sich die individuelle Einschätzung mit der Ideologie der rechtsextremistischen Parteien und der freien Kameradschaften, die hinsichtlich ihrer klassischen Positionen wie Fremdenfeindlichkeit, EU-Ablehnung usw. ein Meinungsdiktat in

18 Als Indikatoren für rechtsextreme Zuschriften sind zu nennen: Grußformeln (z. B. mit germanischem Gruß), Sprachmuster aus der Zeit des Nationalsozialismus (z. B. der Jud Scharon), Einzellexeme wie Reichshauptstadt oder Ironisierungen wie sogenannte $B R D$, Fahnen- und Hochwertwörter des Rechtsextremismus (wie Volk, Reich usw.), extensiver Gebrauch des Attributs deutsch, stark rassistische oder migrantenfeindliche Konzeptualisierungen und Verbalisierungen, auffällige E-Mail-Adressen oder Pseudonyme (wie wotan...@web.de oder ein aufrichtiger Deutscher), Verweise auf Parteimitgliedschaft bzw. Nähe zu NPD oder Republikanern. Indikatoren für linksextreme Zuschriften sind u. a.: Verweise auf einschlägige linksextreme Medien (z. B. Rote Fahne), aber auch Verweise auf die israelische Friedensbewegung, Amnesty International und deren stark globalisierungsund kapitalismuskritische Argumentationen, Kopplung mit extremem Anti-Amerikanismus (Zielperson George W. Bush), Verweise auf Parteimitgliedschaft bzw. Nähe zur Partei Die Linke. 
Deutschland konstatieren und sich selbst als Opfer dieser Zensur gerieren. Um ihre radikale Meinung trotzdem kundzutun, schreiben Rechtsextreme ihre Briefe und E-Mails also im Schutz der Anonymität (bzw. greifen auf Umwegkommunikationen bzw. sprachliche Implizitheit zurück). Insgesamt zeichnet sich damit ab, dass Anonymität (neben den oben genannten Kriterien) ein deutlicher Hinweis darauf ist, dass der Schreiber eine extremistische Position vertritt.

Die analysierten Zuschriften geben in vielfältiger Weise direkte und indirekte Hinweise auf den sozioökonomischen Status ihrer Autoren: Einerseits geben sie sehr häufig selbst explizit Auskünfte über ihren Bildungsstand, ihren Beruf oder ihre Familien- und Lebensumstände. So beginnen viele Zuschriften mit Aussagen wie als $x$-jähriger Renter aus $X$ oder als $x$-jähriger Student habe ich folgende Fragen ... Eine weitere Form der Selbstauskunft bilden ausführliche Schilderungen der eigenen Familiengeschichte oder das Nennen von Interessengebieten oder Beweggründen zum Schreiben (wie z. B. ... beschäftige ich mich als Lehrer schon lange mit der Geschichte der Juden ...; möchte ich meinen Kindern erklären ...; ... für Toleranz und Offenheit einstehend, muss ich ...). In der Regel spielt bei diesen Formen vor allem die positive Selbstdarstellung eine Rolle (vgl. Kap. 11.2). Andererseits liefern formale Merkmale der Zuschriften wie Berufsbezeichnungen (vor allem Pfarrer, Lehrer, Beamter, Kaufmann usw.) oder akademische Titel aber auch Firmenbriefköpfe (z. B. Malerfachbetrieb, Consulting GmbH usw.) und Links auf Websites Hinweise zur Verortung in der Gesellschaftsmitte. Zu erwähnen bleibt, dass die Autoren häufig natürlich auch dezidiert ihre politische Einstellung thematisieren bzw. explizit angeben, keiner (vor allem rechts)extremen Ideologie anzuhängen und vielmehr Teil der ganz „normalen“ Mitte zu sein. Hier wird deutlich, dass auch die Schreiber, die sich an die IBD und den ZJD wenden, ganz verschiedene Kriterien nennen, die sie ihrer Meinung nach als Mitte charakterisieren.

Hinsichtlich des Explizitheitsgrades ist auffällig, dass extreme, explizit (vulgär)antisemitische Zuschriften (vor allem der rechts- und linksextremen Tendenz) eher als E-Mails versendet werden und vom Umfang sehr kurz sind, während vorsichtige, vor allem indirekt und implizit argumentierende Schreiber ausführlicher formulieren und oft eine formal ansprechende Briefform bevorzugen. Viele E-Mails sind lang, d.h. sie umfassen oft mehrere Seiten. Häufig sind Anhänge hinzugefügt; vielfach werden (von den Schreibern aus der Mitte) Kopien an Zeitungsredaktionen und Politiker gesendet.

Im Zeitverlauf nimmt die Zahl der explizit antisemitischen und aggressiven Zuschriften deutlich zu. Explizite Hass- und Drohmails, wie sie ab 2006 zu beob- 
achten sind, kommen in den Jahren 2002 bis 2005 noch nicht bzw. nur selten vor. ${ }^{19}$

\section{Klassifikationskriterien (Variablenset) für die qualitativen Einzeltextanalysen}

Die einzelnen Zuschriften wurden textwissenschaftlich und inhaltsorientiert auf der Basis eines Variablensets nach bestimmten, linguistischen und diskurshistorischen Klassifikationskriterien analysiert. Das Variablenset (vgl. zu den Variablen und ihren jeweiligen Ausprägungen Tabelle 3) setzt sich aus formalen Variablen, wozu u. a. die Identifikationsvariablen („Chiffre und Speicherort“), aber auch Angaben zum Absender („Name, Vorname, Titel, Adresse, Ort, E-Mail-Adresse/ Telefonnummer") und der Umfang der Zuschrift gehören, und Bewertungs- bzw. Valenzvariablen zusammen. ${ }^{20}$

Die Variablen sind in der Mehrzahl nominal skaliert (nur zum Teil waren Mehrfachnennungen möglich, z. B. bei der dominanten Stereotypklasse „tradiert/neu“). Wegen ihrer großen Streubreite wird die Variable ,verbale Auffälligkeiten/Besonderheiten“ offen kodiert, die Kodierer können also ohne Kategorienraster angeben, welche verbalen oder konzeptuellen Muster innerhalb der Zuschrift hervorstechen bzw. dominant sind. Zu den Bewertungsvariablen gehört eine Reihe dichotomer Kriterien, mit denen das Vorkommen bestimmter Strategien oder Manifestationsformen erhoben wird (jeweils Ausprägung „positiv“ und „negativ“ für Auftreten bzw. Fehlen):

Die Variable „NS-Vergleich“ gibt an, ob in der Zuschrift ein oder mehrere NSVergleiche gezogen werden. Bei „Emotion“ wird kodiert, ob in der Zuschrift Emotionen genannt oder ausgedrückt werden (Indikatoren sind emotionsbezeichnende und emotionsausdrückende Lexeme, aber auch para-verbale Signale wie (Farb)Markierungen, Emoticons oder extensiver Gebrauch von Satzzeichen u. Ä.). Zudem wird festgehalten, wenn sich die Schreiber unspezifisch auf Medien beziehen (,indirekter Medienbezug“, z. B. durch Aussagen wie habe ich gelesen, habe ich erfahren usw.), oder wenn sie spezifische Medienberichte nennen, zitieren oder als Anhang beifügen („,konkreter Medienbezug“, z. B. ... habe ich gestern in der Frankfurter Allgemeinen Zeitung den Artikel von ...). Unter „Stereotype ja/nein“ wird die Verbalisierung von antisemitischen Stereotypen erfasst, ein wesentli-

19 Ein Anzeichen dafür, dass sich (wie es u. a. Rensmann 2004 konstatiert hat) die Opportunitätsstrukturen des Antisemitismus tendenziell verändert haben und das Feld des Sagbaren sich ausgeweitet hat.

20 Eine Differenz zwischen Analyse- und Auswahleinheit besteht nicht bzw. diese fallen stets zusammen, weil sich das gesamte Variablenset auf die gesamte Zuschrift bezieht. 
cher Indikator dafür, ob eine Zuschrift verbal-antisemitisch ist oder nicht. Die Variable „Art der Stereotype“ gibt über entsprechende Kürzel die dominante Stereotypform an. ${ }^{21}$ Hierbei wird angegeben, ob die auftretenden Stereotype mehrheitlich als klassisch antisemitisch (KlA; wie z. B. JUDEN = GOTTESMÖRDER, JUDEN = WUCHERER, WELTVERSCHWÖRUNG usw.), Post-Holocaust-antisemitisch ${ }^{22}$ (PHA; Z. B. JUDEN NUTZEN DEN HOLOCAUST AUS, KRITIKTABU usw.) oder anti-israelisch (Anti-I; d. h. auf Israel bezogen wie z. B. ISRAELISCHER SONDERSTATUS, STÖRENFRIED usw.) zu klassifizieren sind. Hier ist eine Mehrfachnennung möglich (z. B. KlA/PHA oder KlA/Anti-I), eine Stereotypenhäufung wird bei „verbale Auffälligkeiten/Besonderheiten“ vermerkt. Die wichtigste Bewertungsvariable ist „,verbalantisemitischer Status“. ${ }^{23}$ Je nach Ausprägung der Stereotyp-Variablen sowie der NS-Vergleich-Variable, und nach Berücksichtigung der im Text verbalisierten Strategien (z. B. Legitimierungsstrategien, Antisemitismus-Abwehr) und der auf den Holocaust zielenden Verantwortungsabwehr oder Schuldrelativierungen sowie der israel-feindlichen Perspektivierungen und Konzeptualisierungen wird geprüft, ob die Zuschrift in ihrer Gesamtheit (bzw. nach ihrer globalen Funktion) als antisemitisch, israel-kritisch oder israel-feindlich $\mathrm{zu}$ klassifizieren ist. Als verbal-antisemitisch werden auch die Texte kodiert, bei denen anti-judaistische Redewendungen und Floskeln (wie Auge um Auge, Zahn um Zahn etc.) argumentativ gegen Juden oder Israelis verwendet werden, oder antisemitische Konzeptualisierungen offensichtlich nicht-intentional geäußert werden (z. B. wenn von Juden als Gästen/Ausländern gesprochen wird, sonst aber eine gänzlich positive und/oder solidarische Bewertung von Juden vorgenommen wird). „Solidarisch“ steht für Zusendungen, in denen keine antisemitischen oder israel-kritischen Konzeptualisierungen und stattdessen Solidaritätsbekundungen ausgedrückt werden. Als philosemitisch werden Zuschriften kodiert, die auf Juden bezogene positive Stereotype enthalten.

Das sprachliche und argumentative Niveau der Zuschriften, also die Eloquenz der verbalen Ausführungen, wird als „normal“ (bzw. „unauffällig“), „bildungsfern“, „,vulgär“ oder „elaboriert“ klassifiziert. So kann unter Umständen

21 Natürlich nur dann, wenn die Variable „Stereotyp ja/nein“ positiv kodiert wurde.

22 Wir lehnen die Terminologie primär versus sekundär antisemitisch ab, da die Termini semantisch keine angemessenen Lesarten liefern und inadäquate Inferenzen aktivieren können (s. hierzu auch Kap. 4.4, Fußnote 53).

23 Die Kodierung richtet sich hier vor allem nach der Zuordnung von judenfeindlichen Stereotypen. Hinzu treten - mit Blick auf den israelbezogenen Antisemitismus - NS-Vergleiche, das Abstreiten des Existenzrechts Israels und doppelte (moralische wie politische) Standards (vgl. u. a. EUMC 2004: 229, Gessler 2004: 10, 15, Heyder et al. 2005: 146 f., Pfahl-Traughber 2007a). 
ein Zusammenhang zwischen Bildungsniveau und der Verbalisierung antisemitischer Stereotype oder NS-Vergleiche aufgedeckt werden.

Als weitere Bewertungsvariable ist die „politische Tendenz“ der Autoren zu nennen. Hier sind neben inhaltlichen Indikatoren auch formale Kontextfaktoren zu beachten, welche auf die politische Haltung oder Tendenz im klassischen Rechts-Mitte-Links-Spektrum hindeuten. In der Regel handelt es sich dabei um explizite oder implizite Selbstauskünfte der Schreiber im Briefkopf/E-MailHeader oder Fließtext. Die jeweilige abgeschwächte bzw. moderate Form dieser Indikatoren spricht für die Kategorien „eher links“ bzw. „eher rechts“. Relevant sind auch das Fehlen solcher Indikatoren und die Angabe von sozialen Schichtungsmerkmalen, die für die sozioökonomische Mitte kennzeichnend sind (z. B. Titel, Berufsbezeichnungen, Bildungsgrad usw.), oder die Parteimitgliedschaft bzw. Nähe zu einer der etablierten Parteien. Hinzu tritt die explizite Selbstattribuierung, zur Gesellschaftsmehrheit oder -mitte zu gehören bzw. Antisemitismus und Rechtsextremismus strikt abzulehnen. 
formale Variablen

\begin{tabular}{|c|c|c|}
\hline Chiffre & ZJD_TT.MM.JJJJ_XXX_Nr. & jeweilige Signatur \\
\hline \multirow[t]{7}{*}{ Art d. Zuschrift } & $\mathrm{E}$ & E-Mail \\
\hline & B & Brief \\
\hline & $\mathrm{Bh}$ & Brief (handschriftlich) \\
\hline & $\mathrm{F}$ & Fax \\
\hline & $\mathrm{Fh}$ & Fax (handschriftlich) \\
\hline & $P$ & Postkarte \\
\hline & $\mathrm{Ph}$ & Postkarte (handschriftlich) \\
\hline Name & {$[\ldots]$} & \\
\hline Vorname & {$[\ldots]$} & \\
\hline Titel & Prof./Dr./Dipl. usw. & akademischer Titel \\
\hline \multirow[t]{2}{*}{ Adresse } & ja & Straße und Wohnort angegeben \\
\hline & nein & Straße und Wohnort nicht angegeben \\
\hline Ort & {$[\ldots]$} & Name vom Ort \\
\hline \multirow[t]{3}{*}{ E-Mail/Tel. } & $\mathrm{T}$ & Telefonnummer angegeben \\
\hline & $\mathrm{E}$ & E-Mail-Adresse angegeben \\
\hline & $\mathrm{E} / \mathrm{T}$ & E-Mail und Tel. angegeben \\
\hline \multirow[t]{3}{*}{ Umfang } & kurz & Text umfasst wenige Zeilen \\
\hline & mittel & Text umfasst weniger als 1 Seite \\
\hline & lang & Text umfasst mehr als 1 Seite \\
\hline \multirow[t]{4}{*}{ Speicherort } & Ordner & Zuschrift liegt papieren vor \\
\hline & $\mathrm{PC}$ & Zuschrift liegt digital vor \\
\hline & PC/Ordner & Zuschrift liegt sowohl papieren als auch digital \\
\hline & & vor \\
\hline
\end{tabular}


Bewertungsvariablen / Valenzvariablen

\begin{tabular}{|c|c|c|}
\hline polit. Tendenz & $\begin{array}{l}\text { r.ex. } \\
\text { Mr } \\
\text { Mitte } \\
\text { Ml } \\
\text { l.ex. } \\
\text { islam } \\
\text { psych }\end{array}$ & $\begin{array}{l}\text { rechtsextrem } \\
\text { eher rechts/rechtskonservativ } \\
\text { gesell. Mitte } \\
\text { eher links } \\
\text { linksextrem } \\
\text { islamistisch } \\
\text { psychopathologisch }\end{array}$ \\
\hline sprachl. Niveau & $\begin{array}{l}\text { norm } \\
\text { elab } \\
\text { bild } \\
\text { vul }\end{array}$ & $\begin{array}{l}\text { normal/unauffällig } \\
\text { elaboriert/hohes sprachliches Niveau } \\
\text { „bildungsfern“/niedriges sprachliches Niveau } \\
\text { vulgär }\end{array}$ \\
\hline antisem. Status & $\begin{array}{l}\text { neutr } \\
\text { sol } \\
\text { phil } \\
\text { ik } \\
\text { eas } \\
\text { ias }\end{array}$ & $\begin{array}{l}\text { neutral (deskriptiv; nicht antisemitisch) } \\
\text { solidarisch } \\
\text { philosemitisch } \\
\text { israel-kritisch (jedoch nicht antisemitisch) } \\
\text { explizit verbal-antisemitisch } \\
\text { implizit verbal-antisemitisch }\end{array}$ \\
\hline Art des Antisem. & $\begin{array}{l}\text { KIA } \\
\text { PHA } \\
\text { IA }\end{array}$ & $\begin{array}{l}\text { klassischer Antisemitismus } \\
\text { Post-Holocaust-Antisemitismus } \\
\text { israelbezogener Antisemitismus }\end{array}$ \\
\hline Stereotyp ja/nein & $\begin{array}{l}\text { ja } \\
\text { nein }\end{array}$ & $\begin{array}{l}\text { judeophobe Stereotype kommen vor } \\
\text { es kommen keine Stereotype vor }\end{array}$ \\
\hline Art der Stereotype & $\begin{array}{l}\text { KIA } \\
\text { PHA } \\
\text { anti-israelisch }\end{array}$ & $\begin{array}{l}\text { klassisch antisemitische Stereotype } \\
\text { Post-Holocaust-antisemitische Stereotype } \\
\text { neue (israelbezogene) Stereotype }\end{array}$ \\
\hline NS-Vgl. & $\begin{array}{l}\text { ja } \\
\text { nein }\end{array}$ & $\begin{array}{l}\text { NS-Vergleiche kommen vor } \\
\text { es kommen keine NS-Vergleiche vor }\end{array}$ \\
\hline Medienbezug & $\begin{array}{l}- \\
\text { ind. MB } \\
\text { konk. MB }\end{array}$ & $\begin{array}{l}\text { keine Medienbezug } \\
\text { indirekter Medienbezug } \\
\text { konkreter Medienbezug (auf Einzelmedium/-text) }\end{array}$ \\
\hline Emotion & $\begin{array}{l}\text { ja } \\
\text { nein }\end{array}$ & $\begin{array}{l}\text { Emotionen werden ausgedrückt/benannt } \\
\text { Emotionen werden nicht ausgedrückt/benannt }\end{array}$ \\
\hline auffällig & {$[\ldots]$} & auffälligste verbale/konzeptuelle Merkmale \\
\hline Sonstiges/Anmerkungen & {$[\ldots]$} & Anmerkungen für Team (z. B. „Textteile fehlen“) \\
\hline
\end{tabular}

Tabelle 3: Übersicht Variablen und Ausprägungen. 
Die Ergebnisse der Textanalysen sind zwar digital erfasst, es wurde jedoch keine computergestützte Analyse durchgeführt, weil diese u. a. keine impliziten Bedeutungskomponenten erfassen kann. Die Analyse richtet sich nach der Äußerungsbedeutung im spezifischen Kontext der jeweiligen Äußerung. So lässt sich der Tendenz entgegenwirken, durch numerische Kodierungen die semantischen und kontextuellen Bedeutungen praktisch zu eliminieren. ${ }^{24}$

\section{Verbal-Antisemitismus: Quantitative Befunde}

Obgleich unsere Korpusanalyse primär qualitativ ausgerichtet ist, wurden auch quantitative Analysen durchgeführt, um z. B. Angaben über signifikante Verteilungsmuster machen zu können.

Bei der Bewertungsvalenz der Zuschriften ist offensichtlich, dass die Meinungsposition der Schreiber in ihrer Mehrheit negativ gegenüber Juden/Judentum ist. 72,6 Prozent aller Zuschriften sind als antisemitisch zu bewerten. Bei den extremistischen Randgruppen sind annähernd alle Zuschriften antisemitisch. Aber auch über ein Drittel der Schreiber der Mitte artikuliert klar antisemitisches Gedankengut (hier bestätigen sich sozialwissenschaftliche Erhebungen, die eine Antisemitismusrate von über 20 Prozent angeben). ${ }^{25}$ Auffällig ist, dass der Anteil für den Zentralrat höher ist als für die Botschaft; die Zuschriften an den ZJD sind häufiger als eindeutig antisemitisch zu klassifizieren als die der Botschaft.

Dem stehen nur neun Prozent neutrale oder legitim kritische Reaktionen entgegen sowie 14 Prozent positive bzw. solidarische Zuschriften. Philosemitische Zuschriften liegen bei 0,4 Prozent. Die Mehrheit der Solidaritätsschreiben ist Teil einer Postkarten-Aktion der Israelischen Botschaft, bei der ca. 230 Sympathiebekundungen eingereicht wurden, oder sie beziehen sich auf das Staatsjubiläum Israels. Darüber hinaus treten solidarische oder positive Zuschriften nur vereinzelt auf. Ab dem Jahr 2007 sind sie aber kontinuierlich im Korpus vorhanden. Neben expliziten Solidaritätsbekundungen wird in diesen Schreiben häufig die deutsche Berichterstattung über Israel als einseitig oder israel-feindlich kritisiert.

Die solidarischen Zuschriften (aus der Mitte der Gesellschaft) richten sich stets an die Botschaft, nicht an den Zentralrat: Uneingeschränkte Solidaritätsschreiben kommen nur in Hinsicht auf Israel vor; Schreiben an den Zentralrat,

24 Vgl. Brosius/Koschel (2001: 202).

25 So gibt z. B. das Pew Research Center (2008) für Deutschland einen Anteil von 25 Prozent Antisemiten an. Der höhere Wert von 32 Prozent könnte u. a. damit erklärt werden, dass soziale Erwünschtheit und Reaktivitätseffekte bei Befragungen deren Ergebnisse beeinflussen und sich daher tendenziell eher geringere Werte ergeben. 
die parallel ein Gedenken an den Holocaust fordern oder befürworten, treten dagegen kaum auf.

Vier Prozent der Schreiben stellen völlig inkohärente und semantisch konfuse Texte dar, die keiner Kategorie zugeordnet werden können. Mehr als die Hälfte der beim ZJD eingegangen Zuschriften ( $n=1.209$, 55,7 Prozent) ist als explizit antisemitisch klassifiziert worden, diese Texte weisen also die Indikatoren für Verbal-Antisemitismus in manifester Form auf, und die Verfasser drücken tradierte judenfeindliche Stereotype (Juden als WUCHERER, LÜGNER, Schuldoder Antisemitismus-Abwehr, Revisionismus etc.) aus. Für das Teilkorpus der Israelischen Botschaft ist dieser Wert mit 25,8 Prozent geringer, dort überwiegen anti-israelische Zuschriften mit 33,9 Prozent, die jedoch oft implizit Stereotypkodierungen erkennen lassen und auf NS-Vergleiche zurückgreifen. Konzeptuelle Verschiebungen und Erweiterungen von tradierten Stereotypen auf aktuelle Negativ-Klischees sind hier frequent zu beobachten (s. Kap. 7).

Für das Stichprobenkorpus 2002 bis 2007 ergibt sich folgende Verteilung für NS-Vergleiche:

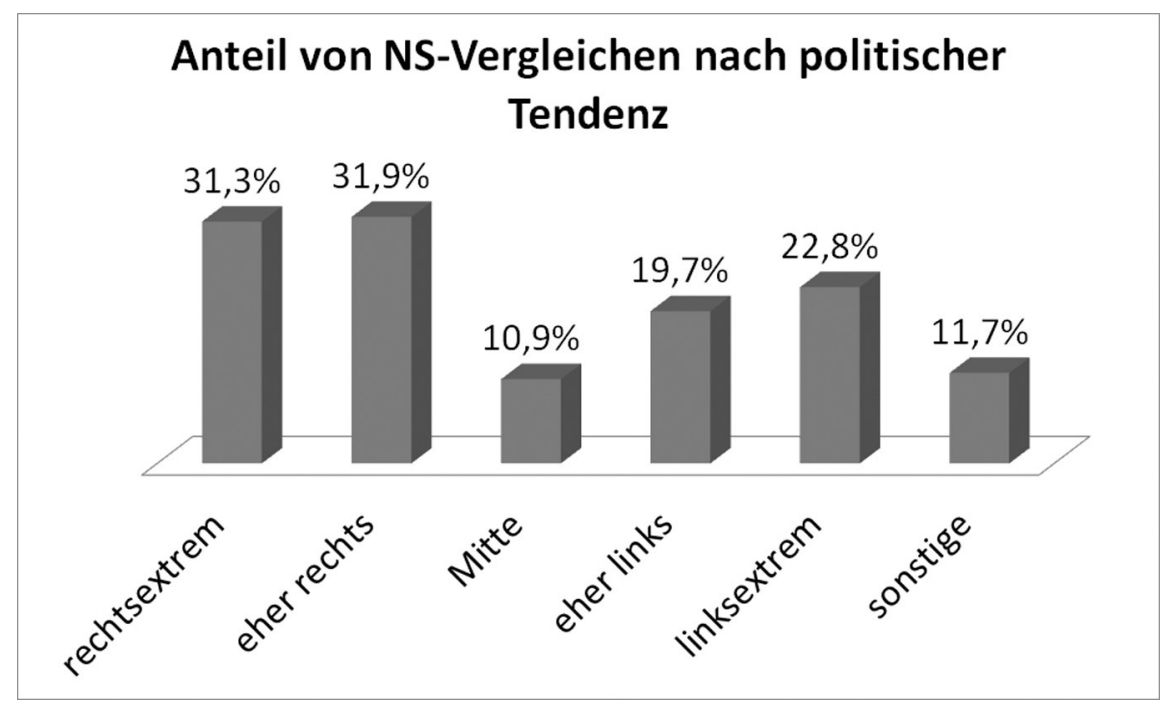

Abbildung 3: Häufigkeit von NS-Vergleichen nach politischer Tendenz.

Die Stichproben für das Material ab 2008 zeigen aber kontinuierlich einen starken Anstieg der NS-Vergleiche seit der Eskalation im Gaza-Streifen; insbesondere ist bei den Schreibern aus der Mitte eine deutliche Zunahme zu verzeichnen: Repräsentative Stichproben innerhalb des neuen Materials bis Ende 2011 deuten hier 
auf einen Prozentsatz von über 30 Prozent hin, d.h. es besteht demzufolge kein Unterschied mehr zwischen Mitte und Rechts- sowie Linksextremen bezüglich der Verwendung solcher inadäquaten Sprachgebrauchsmuster.

\section{Notationskonventionen}

Alle orthographischen und grammatischen Fehler in den Beispielen entsprechen den Originaldokumenten.

Den Konventionen der Kognitionswissenschaft und Linguistik folgend, werden Belegbeispiele vom Text abgesetzt und nummeriert. Im Fließtext erwähnte sprachliche Ausdrücke und Beispiele werden durch Kursivierung hervorgehoben. Konzeptuelle, d. h. mental-geistige Entitäten und Strukturen werden mittels durchgängiger Großschreibung (Kapitälchen) kenntlich gemacht. Bedeutungsparaphrasen, Argumente und Schlussfolgerungen werden in einfache Anführungszeichen gesetzt. 